\title{
Evaluation of antigen-positive toxin-negative enzyme immunoassay results for the diagnosis of toxigenic Clostridium difficile infection
}

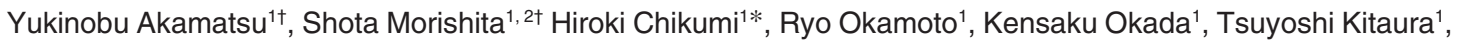 \\ Naomi Miyake $^{3}$, Kosuke Yamaguchi ${ }^{3}$, Masaki Nakamoto ${ }^{1}$, Hisashi Shimohiro ${ }^{4}$, Miyako Takata ${ }^{4}$, Akira Yamasaki ${ }^{3}$, \\ Naoto Burioka ${ }^{4}$, and Eiji Shimizu ${ }^{3}$ \\ ${ }^{1}$ Center for Infectious diseases, Tottori University Hospital, Yonago, Japan, ${ }^{2}$ Division of Clinical Laboratory, Tottori University Hospital, \\ Yonago, Japan, ${ }^{3}$ Division of Medical Oncology and Molecular Respirology, Department of Multidisciplinary Internal Medicine, School of \\ Medicine, Tottori University Faculty of Medicine, Yonago, Japan and ${ }^{4}$ Department of Pathobiological Science and Technology, School of Health \\ Science, Tottori University Faculty of Medicine, Yonago, Japan
}

\begin{abstract}
Clostridium difficile (C. difficile)-associated diarrhea (CDAD) is a challenging nosocomial infectious disease. C. DIFF Quik Chek Complete assay is widely used to detect glutamate dehydrogenase (GDH) antigen and toxin A/B of C. difficile simultaneously. However, the interpretation of GDH positive/toxin negative results is problematic. We performed a retrospective study of patients with GDH positive/toxin negative results to determine the probability of detecting toxigenic C. difficile and its risk factors. Between April 2012 and March 2017, we investigated cultures of fecal specimens followed by toxin detection tests. The clinical histories of patients with and without toxigenic $C$. difficile were compared using univariate- and multivariate-analyses. In total, 2675 patients were examined using C. Diff Quik Chek Complete assay. Among 356 GDH positive/toxin negative patients, cultures were performed in 220 cases and toxigenic $C$. difficile was recovered from $139(63.2 \%)$ specimens. Patients with toxigenic $C$. difficile had significantly lower body mass index than those without. Over half the GDH positive/toxin negative patients were infected with toxigenic C. difficile. Lower BMI was a CDAD risk factor in this patient population. These data can be utilized to initiate isolation and clinical interventions before confirmatory test results are available. J. Med. Invest. 65 : 131 - 135, February, 2018
\end{abstract}

Keywords : C. Diff Quik Chek Complete assay, Clostridium difficile (C. difficile), C. difficile-associated diarrhea (CDAD), GDH positive/toxin negative

\section{INTRODUCTION}

Clostridium difficile (C. difficile) is currently considered to be one of the most formidable and prominent causes of nosocomial infections. $C$. difficile-associated diarrhea (CDAD) might have various symptoms from mild diarrhea to fulminant pseudomembranous colitis, toxic mega-colon, and sepsis. The main cause of this condition is the overwhelming of the normal intestinal flora by the dominant $C$. difficile via the antibiotic turmoil, followed by toxin production by the bacteria. However, the challenge is not only the complications which come after antibiotic exposure, but also the challenges the condition poses to the prevention and control of nosocomial transmission. This is due to the fact that $C$. difficile spores can be shed into the hospital environment by individuals who are infected or colonized. Therefore accurate diagnosis followed by effective therapy in combination with early isolation of the patients is of vital importance.

Various laboratory test are currently available for the detection of $C$. difficile or its toxins (1). The most sensitive diagnostic method, considered the gold standard method for the detection of toxigenic $C$. difficile, is the culturing of the organism under anaerobic conditions with toxin testing of the isolated $C$. difficile. However, it takes several days to get a result and is thus not suitable for the

${ }^{\dagger}$ These authors contributed equally to this work. early diagnosis of $\mathrm{CDAD}$ patients. Polymerase chain reaction (PCR) testing has recently developed highly sensitive and specific methods, however it is still costly and it is difficult to obtain quick results at hospitals where gene amplification machines are not equipped. Additionally, the high sensitivity of the PCR can result in a high proportion of false positive results, as it cannot distinguish CDAD patients from the patients with $C$. difficile asymptomatic carriage. Therefore, various enzyme immunoassay (EIA) based screening methods to detect $C$. difficile glutamate dehydrogenase (GDH) and toxins $\mathrm{A}$ and $\mathrm{B}$ have been developed and are in widespread use (2).

The C. Diff Quik Chek Complete assay is a rapid membrane EIA assay that combines the detection of both GDH and the toxin A and B (3). It has been widely use in hospitals due to its simplicity, rapidity, and cost-effectiveness. Although the performance for the detection for GDH and toxins had been improved compared with other EIA kits (4), the sensitivity and specificity of GDH detection by this kit was reported to be $91.0-100 \%$ and $92.4-94.6 \%$, whereas those for toxin detection were $61.9-78.6 \%$ and $96.9-99.2 \%$, respectively $(3,5,6)$. Because of the discrepancies in the sensitivity between GDH and toxin detection, there is uncertainty regarding clinical decision making for patients with a GDH positive/toxin negative result using this kit. The GDH positive/toxin negative

Received for publication December 21, 2017 ; accepted February 5, 2018.

Address correspondence and reprint requests to Hiroki Chikumi, MD, $\mathrm{PhD}$, Center for Infectious diseases, Tottori University Hospital, 36-1 Nishi-machi, Yonago City, Tottori 683-8504, Japan and Fax : +81-85938-6086. 
results could indicate the presence of toxin producing $C$. difficile or toxin non-producing $C$. difficile, or the lack of $C$. difficile. We therefore evaluated GDH positive/toxin negative cases detected using the C. Diff Quik Chek Complete assay and compared them to the results obtained by conducting anaerobic cultures followed by toxin detection.

\section{MATERIALS AND METHODS}

\section{Study site, stool specimens and study eligibility criteria}

The study was performed in the Tottori University Hospital, a tertiary medical hospital with 40 departments with 697 beds, which is located in the San-in district of West Japan. Liquid or semisolid stool samples obtained from patients hospitalized and submitted for $C$. difficile testing from April 2012 to March 2017 were included in the study. All samples were tested within $24 \mathrm{~h}$ of receipt using the C. Diff Quik Chek Complete assay. Stool samples negative for GDH were not tested further, since the negative predictive value of GDH negative samples ranges between $92.4-100 \%(5,6)$. Samples that tested GDH positive and toxin negative were subsequently analyzed using anaerobic cultures followed by toxin testing of the isolated $C$. difficile. Additionally patients with a GDH positive/toxin negative result were asked to submit another stool sample for additional $C$. difficile culturing.

We registered the results of the first stool sample as one episode and excluded the result of subsequent tests. Additionally, we excluded the samples from the patients who had been hospitalized for less than 2 days because a diagnosis of hospital-acquired $\mathrm{CDAD}$ requires the occurrence of diarrhea at least $48 \mathrm{~h}$ after admission.

This study was conducted in accordance with the Declaration of Helsinki 1975, as revised in 2008 and was approved by the Tottori University Hospital Research Ethics Board (No. 17A079). The requirement for written informed consent was waived by the ethical board.

C. Diff Quik Chek Complete assay and culture of C. difficile followed by toxin testing

All samples were tested using the C. Diff Quik Chek Complete assay (Alere Inc., Waltham, MA, USA) in accordance with the manufacturer's instructions. For culture testing, flesh stool $(1 \mathrm{~mL})$ was incubated with equal amounts of 99\% ethanol (Wako Pure Chemical Industries Ltd., Osaka, Japan) at room temperature for $30 \mathrm{~min}$. Thereafter, 2 to 3 drops of liquid stool were inoculated onto cycloserinecefoxitin-mannitol agar (CCMA) EX (Nissui Pharmaceutical Co. Ltd., Tokyo, Japan). Plates were incubated anaerobically for as long as 3 days at $35^{\circ} \mathrm{C}$ and the suspicious colonies were identified using MALDI Biotyper (Bruker Corporation, Billerica, MA, USA). Colonies identified as $C$. difficile were further tested for toxin positivity using the C. Diff Quik Chek Complete assay.

\section{Data collection}

The gold standard for the diagnosis of toxigenic CDAD in this study was the detection of $C$. difficile by culture followed by the confirmation of toxin production. The patients with GDH positive and toxin negative results using the C. Diff Quik Chek Complete assay were grouped as toxigenic $C$. difficile positive and toxigenic C. difficile negative according to the gold standard testing mechanism. Clinical histories of the patients in each group were collected from medical records. These included demographic characteristics (age, gender) and clinical conditions [body mass index (BMI)], nutritional status indicated by controlling nutritional status (CONUT) score (7), length of hospitalization, antibiotic exposure, medical treatment prior to the diagnosis of $\mathrm{CDAD}$ [insertion of nasogastric tube, urgent admission to intensive care unit (ICU), admission of surgical service], and laboratory test results [total white blood cells, hemoglobin, albumin, blood urea nitrogen (BUN), creatinine, estimated glomerular filtration rates (eGFR)].

\section{Statistical analyses}

To assess the relationship between clinical histories and the presence of toxigenic $C$. difficile in the patients with GDH positive/ toxin negative results using the C. Diff Quik Chek Complete assay, the groups were compared using univariate and multivariate analyses. For univariate analysis, Student's $t$-tests were used to compare continuous variables and Mann-Whitney $U$ tests were used to compare scale or ordinal variables. Chi-squared tests were used to compare proportions for categorical variables. For multivariate analysis, multiple logistic regression analysis was performed. Statistical analyses was performed using IBM SPSS Statistics version 25.0 for Windows (IBM, Chicago, IL, USA) and $P$ values of $<0.05$ were considered statistically significant.

\section{RESULTS}

During the study period, a total of 2675 stool specimens were submitted for $C$. difficile antigen testing. Specimens were first tested using the C. Diff Quik Chek Complete assay. Of all specimens, 234 (8.8\%) were GDH positive/toxin positive (GDH+/ Toxin +$), 356(13.3 \%)$ were GDH positive/toxin negative (GDH+/ Toxin-), and 2085 (77.9\%) were GDH negative/toxin negative (GDH-/Toxin-). Of 356 GDH positive/toxin negative specimens, 136 were excluded from the analysis (11 outpatients and 125 did not meet the study eligibility criteria) and remaining 220 specimens were further analyzed by a $C$. difficile culture followed by toxin testing. Of 220 cultured specimens, toxigenic $C$. difficile and nontoxigenic $C$. difficile were identified in $139(63.2 \%)$ and $60(27.3 \%)$ samples, respectively, while $C$. difficile were not identified in 21 $(9.6 \%)$ samples (Figure 1). The positive predictive value (PPV) of GDH positive/toxin negative test results for CDAD was $63.2 \%$.

We next compared the clinical histories of the toxigenic $C$. difficile positive (139 patients) and negative (81 patients) patients. The toxigenic $C$. difficile-negative patients were the combined group of nontoxigenic $C$. difficile-positive (60 patients) and culture-negative (21 patients) patients. The demographic characteristics, clinical conditions and laboratory test results of the two groups were

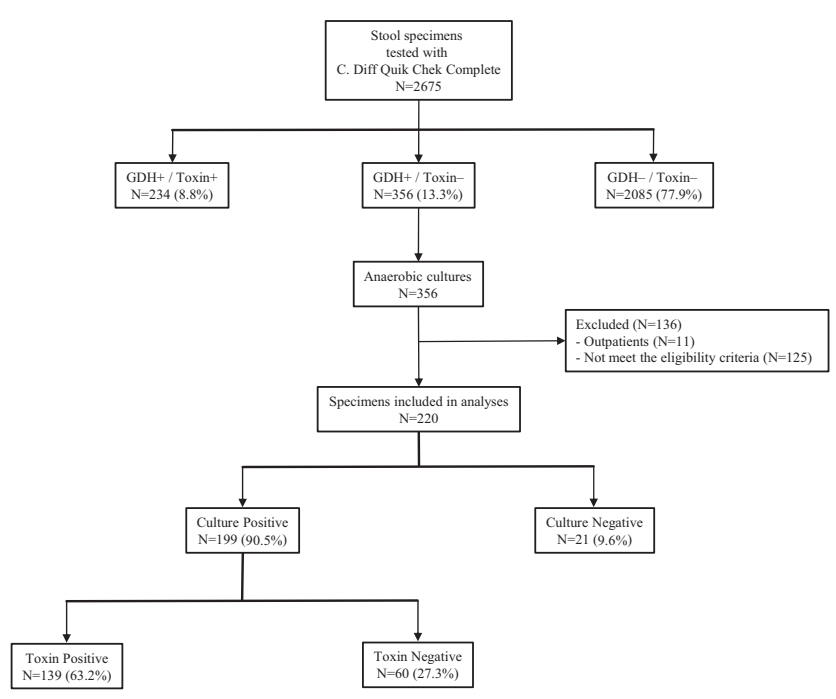

Figure 1. Study enrollment and the testing results 
shown in Table 1. The demographic characteristics did not significantly differ between the groups. However, patients with toxigenic C. difficile had significantly lower BMI compared to those without toxigenic $C$. difficile ( $19.5 \pm 0.4$ vs. $20.9 \pm 0.5, P=0.02)$.

We further investigated the risk factors of toxigenic $C$. difficile detection in the patients with GDH positive/toxin negative results using the C. Diff Quik Chek Complete assay by conducting multivariate logistic regression analysis. As $P$-values of BMI, WBC count and eGFR were $<0.2$ upon univariate analysis, they were included as risk factors in the multivariate analysis. Only lower BMI was a significant risk factor for toxigenic $C$. difficile identification in this patient group [odds ratio (OR) $0.894,95 \%$ confidence interval (CI) $0.828-0.966, P=0.01$ ] (Table 2).

\section{DISCUSSION}

Rapid membrane EIA methods to detect $C$. difficile antigens and toxins are now widely used in clinical settings. Among them, C. Diff Quik Chek Complete assay is one of the most commonly used assay kits, due to its improved sensitivity and specificity. This kit combines the detection of both GDH and toxins A and B, but a discrepancy exists between the sensitivities for detecting GDH and the toxins ; the sensitivity for GDH detection is higher than that for the toxins. As a result, conflicting GDH positive/toxin negative results can be obtained using this kit stemming from three possible causes. One is the presence of toxigenic $C$. difficile, although the toxin cannot be detected due to the lower detection sensitivity for the toxin. The second is the existence of nontoxigenic $C$. difficile, as detected by the kit. The third possibility is the absence of $C$. difficile, with GDH being falsely detected. In this study, we investigated the results of C. Diff Quik Chek Complete assay in our hospital over a five year period, and revealed that $13.3 \%$ of the population investigated had GDH positive/toxin negative results. Among them, $63.2 \%$ of the samples were toxigenic $C$. difficile positive based on the confirmatory culture results followed by toxin testing. These data suggest that there is a large number of patients with GDH positive/ toxin negative results based on the C. Diff Quik Chek Complete assay who are likely true CDAD patients. Additionally, our univariate and multivariate analysis revealed that lower BMI was a risk factor for toxigenic $\mathrm{CDAD}$ in the patients with $\mathrm{GDH}$ positive/toxin negative results.

The possibility of infection with toxigenic $C$. difficile varies significantly from study to study. Based on a PubMed search, various data have been reported regarding the PPV of the C. Diff Quik Chek Complete assay for the detection of toxigenic $C$. difficile among patients with GDH positive/toxin negative results (Table $3)$. The PPV reported in our study (63.2\%) was relatively high compared to the range reported in previous studies (21.7-94.7\%). PPV is strongly influenced by the prevalence of toxigenic $C$. difficile among the study patients. Therefore, most of the samples in our study might have been obtained exclusively from patients with high probabilities of CDAD, such as those who had liquid or semisolid stools during antibiotic therapy.

Our findings provide theoretical evidence to initiate isolation precautions and specific therapy for the patients with GDH positive/ toxin negative results before confirmatory test results are obtained. Although such strategies are generally recommended

Table 1. Clinical backgrounds of the toxigenic $C$. difficile positive and negative patients

\begin{tabular}{|c|c|c|c|c|}
\hline variable & $\begin{array}{l}\text { Toxigenic } C \text {. } \\
\text { difficile } \text { positive } \\
\mathrm{n}=139\end{array}$ & $\begin{array}{l}\text { Toxigenic } C \text {. } \\
\text { difficile negative } \\
\text { (toxin/culture negative) } \\
\mathrm{n}=81\end{array}$ & $P$-value & statistics \\
\hline \multicolumn{5}{|l|}{ Demographic characteristics } \\
\hline Age, mean years $\pm \mathrm{SD}$ & $69.2 \pm 1.5$ & $67.1 \pm 2.4$ & 0.43 & * \\
\hline Sex, no. of patients (Male / Female) & $(83 / 56)$ & $(52 / 29)$ & 0.51 & $\dagger$ \\
\hline \multicolumn{5}{|l|}{ Clinical conditions } \\
\hline BMI $\left(\mathrm{kg} / \mathrm{m}^{2}\right)$, mean $\pm \mathrm{SD}$ & $19.5 \pm 0.4$ & $20.9 \pm 0.5$ & 0.02 & $*$ \\
\hline CONUT score, median (IQR) & $2.5(2-3)$ & $2(1-3)$ & 0.42 & $\ddagger$ \\
\hline Length of hospitalization, mean days $\pm \mathrm{SD}$ & $34.0 \pm 4.5$ & $31.1 \pm 4.3$ & 0.71 & * \\
\hline Antibiotic exposure, no. (\%) of patients & $75(54.0)$ & $47(58.0)$ & 0.56 & $\dagger$ \\
\hline \multicolumn{5}{|c|}{ Medical treatment prior to $\mathrm{CDAD}$, no. (\%) of patients } \\
\hline Nasogastric-tube use & $20(14.4)$ & $16(19.8)$ & 0.35 & $\dagger$ \\
\hline Urgent admission & $10(7.2)$ & $7(8.6)$ & 0.70 & $\dagger$ \\
\hline Admitted surgical service & $34(24.5)$ & $19(23.5)$ & 0.87 & $\dagger$ \\
\hline \multicolumn{5}{|l|}{ Laboratory test results, mean $\pm \mathrm{SD}$} \\
\hline Total WBC count $\left(\times 10^{3} / \mu \mathrm{L}\right)$ & $9.33 \pm 0.57$ & $7.84 \pm 0.52$ & 0.08 & $*$ \\
\hline Total lymphocytes count $\left(/ \mathrm{mm}^{3}\right)$ & $1071 \pm 78$ & $1134 \pm 117$ & 0.64 & $*$ \\
\hline Hemoglobin (g/dL) & $10.1 \pm 0.2$ & $9.9 \pm 0.2$ & 0.39 & $*$ \\
\hline Albumin $(g / d L)$ & $2.7 \pm 0.1$ & $2.6 \pm 0.1$ & 0.80 & * \\
\hline BUN (mg/dL) & $26.2 \pm 2.2$ & $24.3 \pm 1.9$ & 0.53 & $*$ \\
\hline Creatinine $(\mathrm{mg} / \mathrm{dL})$ & $1.5 \pm 0.2$ & $1.6 \pm 0.3$ & 0.58 & * \\
\hline $\mathrm{eGFR}\left(\mathrm{mL} / \mathrm{min} / 1.73 \mathrm{~m}^{2}\right)$ & $89.5 \pm 10.1$ & $70.2 \pm 8.7$ & 0.20 & * \\
\hline
\end{tabular}

*Student's $t$-test, $†$ Chi-squared test, $¥$ Mann-Whitney $U$ test.

Data are number, median (IQR, interquartile range) or mean \pm SD.

CONUT score, controlling nutritional status score (7) : the automatic assessment of nutritional status based on 3 serum parameters : albumin, hemoglobin and total lymphocyte count. Nutritional status was classifies as follows : normal, $0-1$; light, $2-4$; moderate, $5-8$; sever, 9-12.

BMI, body mass index ; BUN, blood urea nitrogen ; eGFR, estimated glomerular filtration rate. 
Table 2. Odds ratios for toxigenic $C$. difficile existence according to various patients' characteristics

\begin{tabular}{llll}
\hline variable & Odds ratio & $\begin{array}{l}95 \% \text { Confidence } \\
\text { interval }\end{array}$ & $P$-value \\
\hline BMI $\left(\mathrm{kg} / \mathrm{m}^{2}\right)$ & 0.894 & $0.828-0.966$ & 0.01 \\
Total $\mathrm{WBC}$ count & 1.039 & $0.981-1.100$ & 0.20 \\
eGFR $\left(\mathrm{mL} / \mathrm{min} / 1.73 \mathrm{~m}^{2}\right)$ & 1.002 & $0.996-1.007$ & 0.52 \\
\hline
\end{tabular}

(8), it is difficult to implement these practices without definitive evidence of these recommendations, as the isolation precautions for CDAD patients are costly and are resource intensive for hospitals (i.e. require isolation-rooms). Therefore, similar investigations in every hospital are of vital importance.

In this study we additionally found that low BMI was a possible risk factor for the development of toxigenic CDAD among those with GDH positive/toxin negative results using the C. Diff Quik Chek Complete assay. To our knowledge this is the first study to investigate risk factors in this patient population. There is limited information in the literature on BMI of patients who harbor toxigenic $C$. difficile. One study reported a CDAD case that was successfully treated with fecal microbiota transplantation and showed weight gain after receiving stools (9). Another study indicated that low BMI is related to impaired immune function (10), and immunosuppression is one of the risk factors of CDAD (11). Therefore, the observed association between lower BMI and toxigenic $C$. difficile in the present study may be mediated by factors that influence the microbiome, or impaired immunological functions of patients with low BMI. Future studies are warranted to identify the factors responsible for the association between low BMI and existence of toxigenic $C$. difficile.

It has been previously reported that antibiotic use (12), advanced age, hospitalization, severe illness, gastric acid suppression, feeding tubes, gastrointestinal surgery, and cancer chemotherapy are risk factors for $\mathrm{CDAD}(11,13-16)$. Our results differed from those from previous studies; previous antibiotic use which is the most common risk factor for CDAD was not identified in our study.
This is due to the fact that we focused on patients with GDH positive/toxin negative results based on the C. Diff Quik Chek Complete assay in this study rather than the general patient population. Most of our study patients possibly had already been prescribed antibiotics, because CDAD was suspected when the stool samples were collected. For the same reason, previously reported risk factors for $\mathrm{CDAD}$ in the general population might be not be identified as risk factors in specific subgroups of patients.

Our study was limited by the fact that it was a retrospective study performed in a single tertiary hospital. However, to our knowledge, this was the second largest study to have ever investigated this topic (Table 3). Additionally, as this was a retrospective study, future prospective studies are needed to confirm our findings.

In conclusion, a substantial proportion of the patients with GDH positive/toxin negative results using the C. Diff Quik Chek Complete assay are infected with toxigenic $C$. difficile and are therefore considered to have true $\mathrm{CDAD}$. This was the case if the specimens were taken from adequate patients such as patients with liquid or semisolid stool after antibiotic use. Investigation of such patients in each hospital provides the theoretical background for the implementation of isolation and therapy strategies for these individuals. Low BMI is an additional risk factor which can be used to identify the patients with true $\mathrm{CDAD}$ in this patient population. These data will helpful to adopt pragmatic strategies for the management of these patients before confirmatory test results are available in each hospital.

\section{DISCLOSURE}

The authors declare no conflict of interest.

\section{ABBREVIATIONS}

C. difficile, Clostridium difficile; $\mathrm{CDAD}, C$. difficile -associated diarrhea ; GDH, glutamate dehydrogenase ; BMI, body mass index ; WBC, white blood cell ; PCR, polymerase chain reaction; EIA, enzyme immunoassay ; CCMA, cycloserine-cefoxitin-mannitol agar ; CONUT, controlling nutritional status ; ICU, intensive care

Table 3. Summary of articles reporting the prevalence of toxigenic C. difficile existence among the GDH positive/toxin negative samples judged by C. Diff Quik Check Complete assay

\begin{tabular}{lllllll}
\hline Author & Year & Country & Reference methods & $\begin{array}{l}\text { Number of Samples } \\
\text { tested by the assay }\end{array}$ & $\begin{array}{l}\text { Number of GDH } \\
\text { positive/toxin } \\
\text { negative (\%) }\end{array}$ & $\begin{array}{l}\text { Number of } \\
\text { Toxigenic } C \text {. difficile } \\
\text { detected (\%) }\end{array}$ \\
\hline Quinn CD et al. (17) & 2010 & USA & PCR & 174 & $23(13.2)$ & $5(21.7)$ \\
Sharp SE et al. (18) & 2010 & USA & PCR and culture & 284 & $27(9.5)$ & $15(55.5)$ \\
Swindells J et al. (19) & 2010 & UK & PCR and culture & 150 & $11(7.3)$ & $8(72.7)$ \\
Kawada M et al. (6) & 2011 & Japan & Culture & 60 & $9(15)$ & $6(66.6)$ \\
Orellana-Miguel MA et al. (20) & 2012 & Spain & Culture & 970 & $60(6.18)$ & $25(41.7)$ \\
Culbreath K et al. (21) & 2012 & USA & PCR & 4321 & $566(13.1)$ & $342(60.4)$ \\
Vasoo S et al. (22) & 2014 & USA & PCR & 192 & $25(13.02)$ & $10(40)$ \\
Alcala L et al. (23) & 2015 & Spain & PCR and culture & 979 & $103(10.5)$ & $49(47.5)$ \\
Johansson K et al. (24) & 2016 & Sweden & PCR and culture & 419 & $36(8.59)$ & $17(47.2)$ \\
Seo JY et al. (25) & 2016 & Korea & PCR and culture & 191 & $19(9.9)$ & $18(94.7)$ \\
Collins DA et al. (26) & 2017 & Indonesia & Culture & 340 & $54(15.0)$ & $21(38.9)$ \\
Kosai K et al. (27) & 2017 & Japan & PCR and culture & 118 & $36(30.5)$ & $19(52.7)$ \\
This study & 2017 & Japan & Culture & 2675 & $356(13.3)$ & $139(63.2)$ \\
\hline
\end{tabular}

*The percentages were the frequency of toxigenic $C$. difficile existence among the samples with GDH positive/toxin negative judged by C. Diff Quik Check Complete assay. 
unit ; BUN, blood urea nitrogen ; eGFR, estimated glomerular filtration rates ; SD, standard deviation ; PPV, positive predictive value

\section{REFERENCE}

1. Bartlett JG, Gerding DN : Clinical recognition and diagnosis of Clostridium difficile infection. Clin Infect Dis 46 Suppl 1 : S12-18, 2008

2. Martinez-Melendez A, Camacho-Ortiz A, Morfin-Otero R, Maldonado-Garza HJ, Villarreal-Trevino L, Garza-Gonzalez $\mathrm{E}$ : Current knowledge on the laboratory diagnosis of Clostridium difficile infection. World J Gastroenterol 23 : 15521567, 2017

3. Sharp SE, Ruden LO, Pohl JC, Hatcher PA, Jayne LM, Ivie WM : Evaluation of the C.Diff Quik Chek Complete Assay, a new glutamate dehydrogenase and $\mathrm{A} / \mathrm{B}$ toxin combination lateral flow assay for use in rapid, simple diagnosis of clostridium difficile disease. J Clin Microbiol 48 : 2082-2086, 2010

4. Crobach MJ, Dekkers OM, Wilcox MH, Kuijper EJ : European Society of Clinical Microbiology and Infectious Diseases (ESCMID) : data review and recommendations for diagnosing Clostridium difficile-infection (CDI). Clin Microbiol Infect $15: 1053-1066,2009$

5. Kim H, Kim WH, Kim M, Jeong SH, Lee K : Evaluation of a rapid membrane enzyme immunoassay for the simultaneous detection of glutamate dehydrogenase and toxin for the diagnosis of Clostridium difficile infection. Ann Lab Med 34 : 235239, 2014

6. Kawada M, Annaka M, Kato H, Shibasaki S, Hikosaka K, Mizuno H, Masuda Y, Inamatsu T : Evaluation of a simultaneous detection kit for the glutamate dehydrogenase antigen and toxin $\mathrm{A} / \mathrm{B}$ in feces for diagnosis of Clostridium difficile infection. J Infect Chemother 17 : 807-811, 2011

7. Ignacio de Ulibarri J, Gonzalez-Madrono A, de Villar NG, Gonzalez P, Gonzalez B, Mancha A, Rodriguez F, Fernandez $\mathrm{G}$ : CONUT : a tool for controlling nutritional status. First validation in a hospital population. Nutr Hosp $20: 38-45,2005$

8. Bignardi GE, Hill K, Berrington A, Settle CD : Two-stage algorithm for Clostridium difficile : glutamate-dehydrogenasepositive toxin-negative enzyme immunoassay results may require further testing. J Hosp Infect 83 : 347-349, 2013

9. Alang N, Kelly CR : Weight gain after fecal microbiota transplantation. Open Forum Infect Dis $2:$ ofv004, 2015

10. Dobner J, Kaser S : Body mass index and the risk of infection from underweight to obesity. Clin Microbiol Infect 24 : 24-28, 2018

11. Loo VG, Bourgault AM, Poirier L, Lamothe F, Michaud S, Turgeon N, Toye B, Beaudoin A, Frost EH, Gilca R, Brassard P, Dendukuri N, Beliveau C, Oughton M, Brukner I, Dascal A : Host and pathogen factors for Clostridium difficile infection and colonization. N Engl J Med 365 : 1693-1703, 2011

12. Thomas C, Stevenson M, Riley TV : Antibiotics and hospitalacquired Clostridium difficile-associated diarrhoea : a systematic review. J Antimicrob Chemother 51 : 1339-1350, 2003

13. Kyne L, Sougioultzis S, McFarland LV, Kelly CP : Underlying disease severity as a major risk factor for nosocomial Clostridium difficile diarrhea. Infect Control Hosp Epidemiol 23 : 653659, 2002

14. Dial S : Risk of Clostridium difficile diarrhea among hospital inpatients prescribed proton pump inhibitors : cohort and case-control studies. Canadian Medical Association Journal
$171: 33-38,2004$

15. Bliss DZ, Johnson S, Savik K, Clabots CR, Willard K, Gerding $\mathrm{DN}$ : Acquisition of Clostridium difficile and Clostridium difficile-associated diarrhea in hospitalized patients receiving tube feeding. Ann Intern Med 129 : 1012-1019, 1998

16. Kamthan AG, Bruckner HW, Hirschman SZ, Agus SG : Clostridium difficile diarrhea induced by cancer chemotherapy. Arch Intern Med 152 : 1715-1717, 1992

17. Quinn CD, Sefers SE, Babiker W, He Y, Alcabasa R, Stratton CW, Carroll KC, Tang YW : C. Diff Quik Chek complete enzyme immunoassay provides a reliable first-line method for detection of Clostridium difficile in stool specimens. J Clin Microbiol 48 : 603-605, 2010

18. Sharp SE, Ivie WM, Buckles MR, Coover DM, Pohl JC, Hatcher PA : A simple 3-step algorithm for improved laboratory detection of Clostridium difficile toxin without the need for tissue culture cytotoxicity neutralization assays. Diagn Microbiol Infect Dis 64 : 344-346, 2009

19. Swindells J, Brenwald N, Reading N, Oppenheim B : Evaluation of diagnostic tests for Clostridium difficile infection. J Clin Microbiol 48 : 606-608, 2010

20. Orellana-Miguel MA, Alcolea-Medina A, Barrado-Blanco L, Rodriguez-Otero J, Chaves-Sanchez F : Algorithm proposal based on the C. Diff Quik Chek Complete ICT device for detecting Clostridium difficile infection. Enferm Infecc Microbiol Clin $31: 97-99,2013$

21. Culbreath K, Ager E, Nemeyer RJ, Kerr A, Gilligan PH : Evolution of testing algorithms at a university hospital for detection of Clostridium difficile infections. J Clin Microbiol 50 : 3073-3076, 2012

22. Vasoo S, Stevens J, Portillo L, Barza R, Schejbal D, Wu MM, Chancey C, Singh K : Cost-effectiveness of a modified twostep algorithm using a combined glutamate dehydrogenase/ toxin enzyme immunoassay and real-time PCR for the diagnosis of Clostridium difficile infection. J Microbiol Immunol Infect $47:$ 75-78, 2014

23. Alcala L, Reigadas E, Marin M, Fernandez-Chico A, Catalan P, Bouza E : Comparison of GenomEra C. difficile and Xpert C. difficile as confirmatory tests in a multistep algorithm for diagnosis of Clostridium difficile infection. J Clin Microbiol 53 : 332-335, 2015

24. Johansson K, Karlsson H, Noren T: Clostridium difficile infection diagnostics - evaluation of the C. DIFF Quik Chek Complete assay, a rapid enzyme immunoassay for detection of toxigenic C. difficile in clinical stool samples. APMIS 124 : 1016-1020, 2016

25. Seo JY, Jeong JH, Kim KH, Ahn JY, Park PW, Seo YH : Laboratory diagnosis of Clostridium difficile infection : Comparison of Techlab C. diff Quik Chek Complete, Xpert C. difficile, and multistep algorithmic approach. J Clin Lab Anal 31,2017

26. Collins DA, Gasem MH, Habibie TH, Arinton IG, Hendriyanto P, Hartana AP, Riley TV : Prevalence and molecular epidemiology of Clostridium difficile infection in Indonesia. New Microbes New Infect 18:34-37, 2017

27. Kosai K, Iwanaga Y, Akamatsu N, Okada Y, Kaku N, Uno N, Morinaga Y, Hasegawa H, Miyazaki T, Izumikawa K, Mukae $\mathrm{H}$, Yanagihara K : Performance evaluation of the Verigene ${ }^{\circledR}$ Clostridium difficile nucleic acid test, an automated multiplex molecular testing system for detection of C. difficile toxin. J Infect Chemother $23: 674-677,2017$ 